Revista de Metalurgia

Julio-Septiembre 2018, 54 (3), e127

ISSN-L: 0034-8570

https://doi.org/10.3989/revmetalm.127

\title{
REVISION
}

\section{Evolución y proceso de fabricación de imanes "NEO" aplicados a motores de vehículos eléctricos}

\author{
Victoria Abad $^{\bowtie}$, Jesús Sagredo \\ Universidad de Burgos, Departamento de Ingeniería Electromecánica, Hospital del Rey s/n - 09001 Burgos, España \\ ${ }^{\square}$ Autor para la correspondencia: vaspol@ubu.es
}

Enviado: 9 Junio 2017; Aceptado: 21 Junio 2018; Publicado on-Line: 13 Septiembre 2018

RESUMEN: Tras el desarrollo de los imanes Nd-Fe-B o "Neo", éstos son ahora componentes esenciales en muchos campos de la tecnología debido a su capacidad para proporcionar un fuerte flujo magnético. Existen dos técnicas bien establecidas para la fabricación de imanes de tierras raras: a) el uso de la metalurgia de polvo para obtener cuerpos magnéticos de alto rendimiento, anisotrópicos, totalmente densos; y b) el proceso de hilado por fusión o HDDR (hidrogenación, decrepitación, desorción y recombinación) se usa ampliamente para producir polvos magnéticos para imanes aglomerados. En la industria de los imanes de Nd-Fe-B sinterizados, la producción total ha aumentado y su aplicación dominante es principalmente motores y en particular, su uso para automóviles híbridos es una de las aplicaciones más atractivas. También se han utilizado imanes aglomerados para motores pequeños, los estudios de nanocompuestos y de imanes Sm-Fe-N se han generalizado. Este artículo revisa el estado actual y las tendencias futuras en la investigación de imanes permanentes.

PALABRAS CLAVE: Aglomerado; Imanes permanentes; Sinterizado; Tierras raras

Citar como/Citation: Abad, V.; Sagredo, J. (2018). "Evolución y proceso de fabricación de imanes "NEO" aplicados a motores de vehículos eléctricos". Rev. Metal. 54(3): e127. https://doi.org/10.3989/revmetalm.127

\begin{abstract}
Evolution and process of manufacture of "NEO" magnets applied to motors of electric vehicles. After the development of $\mathrm{Nd}-\mathrm{Fe}-\mathrm{B}$ magnets, rare-earth magnets are now essential components in many fields of technology, because of their ability to provide a strong magnetic flux. There are two, well-established techniques for the manufacture of rare earth magnets: powder metallurgy is used to obtain high-performance, anisotropic, fully dense magnet bodies; and the melt-spinning or HDDR (hydrogenation, disproportionation, desorption and recombination) process is widely used to produce magnet powders for bonded magnets. In the industry of sintered $\mathrm{Nd}-\mathrm{Fe}-\mathrm{B}$ magnets, the total amount of production has increased and their dominant application has been changed to motors. In particular, their use for motors in hybrid cars is one of the most attractive applications. Bonded magnets have also been used for small motors, and the studies of nanocomposite and $\mathrm{Sm}-\mathrm{Fe}-\mathrm{N}$ magnets have become widespread. This paper reviews the current status and future trends in the research of permanent magnets.
\end{abstract}

KEYWORDS: Bonded; Permanent magnet; Rare earth; Sintered

ORCID: Victoria Abad(https://orcid.org/0000-0001-6613-8123); Jesús Sagredo(https://orcid.org/0000-0003-1767-4549)

Copyright: (C) 2018 CSIC. Este es un artículo de acceso abierto distribuido bajo los términos de la licencia de uso y distribución Creative Commons Reconocimiento 4.0 Internacional (CC BY 4.0). 


\section{INTRODUCCIÓN}

Desde que los vehículos eléctricos (EV) entraron con fuerza en el mercado hace 20 años, el uso de imanes de tierras raras se ha hecho imprescindible en ellos, especialmente los de neodimio-hierro-boro, denominados "NEO" o NdFeB. La aplicación de este tipo de materiales en los motores eléctricos viene de la sustitución lógica por los devanados del rotor eliminando a su vez la fuente de excitación como es el caso del utilizado en el exitoso Toyota Prius.

Los elevados valores de producto de energía que presentan estos imanes han permitido conseguir motores eléctricos compactos y con máximos de valores de densidad de par y potencia. Esta tendencia ha permanecido imparable, pero el continuo ascenso de los precios de estos materiales hasta 2012 y la especial situación coyuntural, política y de abastecimiento que sufren, hacen replantear esta constitución. Aunque los precios han disminuido recientemente, la preocupación de los fabricantes de automóviles continúa. Además, se suman los problemas de abastecimiento que actualmente existen por las restricciones impuestas por China, el único suministrador, lo que impulsa a buscar su reemplazo urgente. A esto hay que sumarle la ingente contaminación asociada a la explotación y procesado de estos materiales, lo que afianza la búsqueda de materiales alternativos (DOE, 2011).

Existen dos técnicas claramente establecidas para la fabricación de imanes de tierras raras: a) la metalurgia de polvo se utiliza para obtener cuerpos magnéticos de alto rendimiento, anisotrópicos, totalmente densos; y b) el proceso de hilado por fusión o HDDR (hidrogenación, desproporción, desorción y recombinación) que se usa ampliamente para producir polvos magnéticos para imanes aglomerados.

Aunque son necesarios en muchos campos, su aplicación predominante ha cambiado hacia los motores y, en particular, su uso para motores en automóviles híbridos. Se han generalizado los estudios de nanocompuestos y de imanes Sm-Fe-N, además de comenzar a aplicarse imanes aglomerados para motores pequeños.

\section{CLASIFICACIÓN DE IMANES PERMANENTES}

Según los materiales utilizados los imanes se pueden clasificar en: Ferritas, Alnicos, Samario-cobalto y NdFeB. En la Fig. 1 se muestra la evolución temporal de los imanes respecto a su característica más importante, el producto de energía $\left(\mathrm{BH}_{\max }\right)$ y una comparativa del volumen requerido de cada tipo de imán para obtener la misma energía magnética. Puede apreciarse el destacado rendimiento de los imanes $\mathrm{NdFeB}$ respecto del resto.

\subsection{Ferritas}

Las ferritas son materiales cerámicos ferromagnéticos compuestos por hierro, cobalto y bario con buenas propiedades magnéticas, entre otras, alta permeabilidad magnética. Tradicionalmente se han utilizado para la construcción de núcleos magnéticos e imanes permanentes.

Si se analiza la producción por toneladas constituyen con diferencia la mayor producción de imanes permanentes. Los minerales son baratos en mina, pero presentan bajos valores de producto de energía de tal forma que actualmente sea imposible su aplicación en las aplicaciones de interés.

Ya en los años 50 se probó a hacer sustituciones con estroncio y bario en los óxidos de ferrita, pero sin mucho éxito Al contrario que en los imanes de tierras raras, en las ferritas la coercitividad aumenta con la temperatura, con el valor de $\beta$ más positivo.

Uno de los principales problemas que presentan las ferritas es la tendencia a la desgamentización a bajas temperaturas, aunque se ha mejorado con la adición de cobalto y lantano (Deshpande, 2003).

\subsection{Alnicos}

Estos imanes permanentes forman una familia de aleaciones cuyos componentes principales son: el hierro, el aluminio, el níquel y el cobalto, aunque pueden estar incluidos en cantidades menores otros elementos como cobre y titanio.

Los grados de estos materiales varían con el contenido de cobalto, que va de cero (Alnico 3) a 40\% (Alnico 8).

Los grados inferiores $(2,3$ y 4$)$ son isotrópicos, lo que implica propiedades magnéticas iguales en todas las direcciones, su producción es relativamente baja. Los grados anisotrópics (Alnico 5, 6, 8 y 9) están diseñados para producir un alto rendimiento magnético en una dirección especificada. Las aleaciones anisótropas en general tienen una mayor capacidad magnética. La orientación se consigue durante el tratamiento térmico, mediante el enfriamiento del producto a partir de una temperatura de aproximadamente $1093^{\circ} \mathrm{C}($ Emagnets UK, 2017) a una velocidad controlada, dentro de un campo magnético que se ajusta a la dirección preferida de la magnetización, de este modo se obtiene un material de precipitación que está formado por hierro y cobalto precipitados en una matriz rica en Ni y Al.

Los alnicos presentan buena resistencia a la corrosión, tienen alta remanencia y podrían igualar a los imanes de tierras raras si se mejorara su valor de $H_{C}$. Tienen un alto punto de fusión y, en general, buen comportamiento a altas temperaturas porque son el resultado de la fuerte tendencia hacia el orden, debido a los enlaces intermetálicos. Son también uno de los imanes más estables si se manejan adecuadamente. 


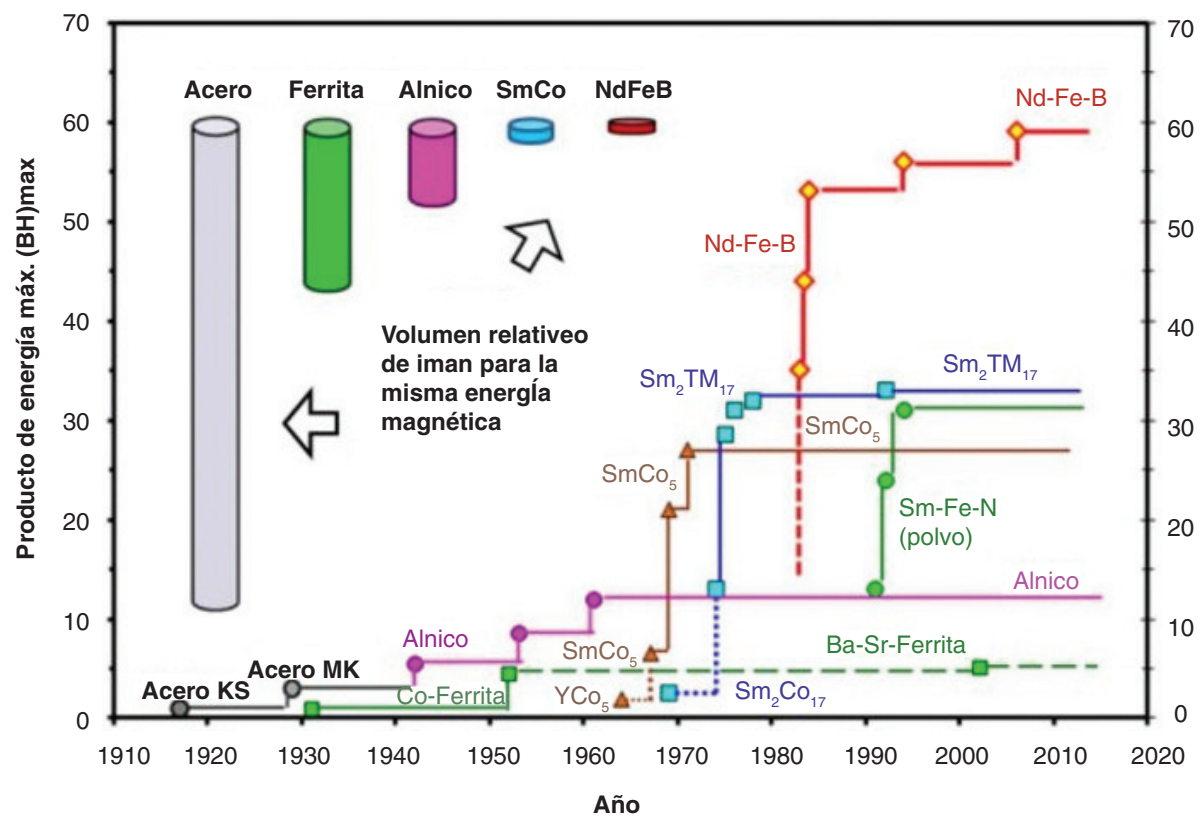

Figura 1. Productos de energía típicos de los distintos tipos de imanes (Keisan, 2016).

Sus componentes se consiguen fácilmente, y su precio es relativamente bajo (alrededor de $44 \$ / \mathrm{kg}$ ) a pesar del alto coste del cobalto.

Por estas propiedades los imanes de Alnico han sido seleccionados por Ames Laboratory como buenos candidatos para obtener imanes sin tierras raras para todas aquellas aplicaciones que requieren temperaturas por encima de los $180^{\circ} \mathrm{C}$.

A nivel de laboratorio se está produciendo gas atomizado, consiguiendo un grano isotrópico fino de alnico denominado 8H (Anderson, 2012a), de resultados esperanzadores.

\subsection{Samario Cobalto}

Estos imanes fueron desarrollados a principio de la década de 1970. Están constituidos por una aleación de hierro, cobalto y una tierra rara ligera: el samario. Son de manera general el segundo tipo más fuerte de imanes, pero menos fuertes que los imanes de neodimio, en cambio tienen mayores temperaturas de trabajo $\left(300{ }^{\circ} \mathrm{C}\right)$ y relativamente grandes coercitividades, que son ventajas insustituibles. Son frágiles, y propensos a agrietarse, pero en cambio no tienen problemas de corrosión. Los valores de los coeficientes de temperatura $\alpha$ y $\beta$ (relación $\mathrm{B} / \mathrm{t}^{\mathrm{a}}$ y $\mathrm{H} / \mathrm{t}^{\mathrm{a}}$ ) son inmejorables. El coste de estos imanes es elevado por los procesos de refinamiento, pero especialmente por el coste de los productos de las minas. Los precios rondan $180 \$ / \mathrm{kg}$, cada vez más próximos a los $150 \$ / \mathrm{kg}$ de los de neodimio sinterizados (Zhang et al., 2012; Boldea et al., 2014; Abad y Sagredo, 2018).
Hay dos familias de materiales de SmCo (Liu, 2010); la serie SmCo 5 ofrece una fácil magnetización en campos moderados y la mejor resistencia a la corrosión de todos los imanes de tierras raras. Los imanes de la otra serie, $\mathrm{Sm}_{2} \mathrm{Co}_{17}$ (Fessler, 2011), muestran el rendimiento magnético más alto a temperaturas elevadas. Se pueden obtener mayoritariamente sinterizados y también, aunque en menor cantidad, como aglomerados.

Como resumen, y antes de profundizar más en los imanes NEO, la Tabla 1 muestra los valores típicos de las características magnéticas de los distintos tipos de imanes. La $t^{\mathrm{a}}$ de Curie es aquella a la que los imanes se degradan magnéticamente.

\subsection{Neodimio-hierro-boro "neos"}

La ventaja de esta aleación es que sus principales componentes ( $\mathrm{Fe}$ y $\mathrm{Nd}$ ) son fáciles de obtener en mina, son baratos y hay reservas más abundantes que las de los imanes de tipo SmCo usados hasta su desarrollo, lo que permitió una reducción significativa de costos de materia prima.

La mezcla de tierras raras con metales de transición forma compuestos intermetálicos que permiten valores elevados de anisotropía y, por tanto, productos de energía elevados. Son, con diferencia, los imanes más potentes conocidos hasta el momento, como se muestra en la Fig. 1.

Los imanes NEO convencionales se componen de tres fases: a) $\mathrm{Nd}_{2} \mathrm{Fe}_{14} \mathrm{~B}$ (composición estequiométrica), b) fase Nd-rica y c) B-rica. La combinación en la proporción adecuada entre las tres fases, junto con 
TABla 1. Características magnéticas típicas de los distintos tipos de imanes (Trout, 2015; Vaimann et al., 2013)

\begin{tabular}{lcccc}
\hline Tipo & $\mathbf{B}_{\mathbf{r}}(\mathbf{k G})$ & $\mathbf{H}_{\mathbf{C}}(\mathbf{O e})$ & $\mathbf{B H}_{\max }(\mathbf{M O e})$ & $\mathbf{t}^{\mathbf{a}} \mathbf{C u r i e}\left({ }^{\circ} \mathbf{C}\right)$ \\
\hline Ferrita cerámica & 4 & 3.300 & 3,8 & 460 \\
Alnico 5 & 12,5 & 640 & 5.5 & 890 \\
Sm-1-5 & 9,0 & 30.000 & 20 & 727 \\
Sm-Co 2-17 & 10,4 & 25.000 & 26 & 825 \\
NdFeB (sinterizado) & 13,4 & 50.000 & 52 & 310 \\
NdFeB (aglomerado) & 7,0 & 9.000 & 18 & 360 \\
\hline
\end{tabular}

las mejoras en las técnicas de fabricación, es lo que les proporciona tan buenas características magnéticas.

El principal inconveniente que presentan es su dependencia con la temperatura, con un valor de temperatura de Curie de tan solo $312^{\circ} \mathrm{C}$ y una temperatura máxima de trabajo de 125 a $150{ }^{\circ} \mathrm{C}$. La adición de disprosio mejora en gran manera este problema $\mathrm{y}$, además, mejora la coercitividad y el producto de energía. La Fig. 2 muestra cómo varían las propiedades de los imanes con la adición de disprosio, y cómo se establece una clasificación comercial estándar que relaciona el compromiso entre este valor y la máxima temperatura de trabajo. En la parte superior de la figura se indican los usos típicos de cada clasificación.

Otro problema que presentan estos imanes es su baja resistencia a la corrosión, que hace necesario aplicar algún tipo de tratamiento superficial tras su conformación.
Por ejemplo, en un imán N38SH, cuyas características magnéticas aparecen en la Fig. 3, la letra $\mathbf{N}$ indica que es $\mathrm{NdFeB}, \mathbf{3 8}$ es el valor de energía (en MGOe) y $\mathrm{SH}$ la máxima temperatura que soporta, que es $150{ }^{\circ} \mathrm{C}$.

\section{PROCESOS DE FABRICACIÓN DE IMANES NEO}

A continuación, se indican las posibilidades de fabricación de los tipos de imanes neo: sinterizados y aglomerados. Las principales características que se busca en un imán son las magnéticas, pero existen otras también importantes, bien de tipo físico que influyen en las posibilidades de fabricación que ofrece el material, bien otras de tipo económico que incluyen aspectos tan relevantes como el coste o la disponibilidad temporal.

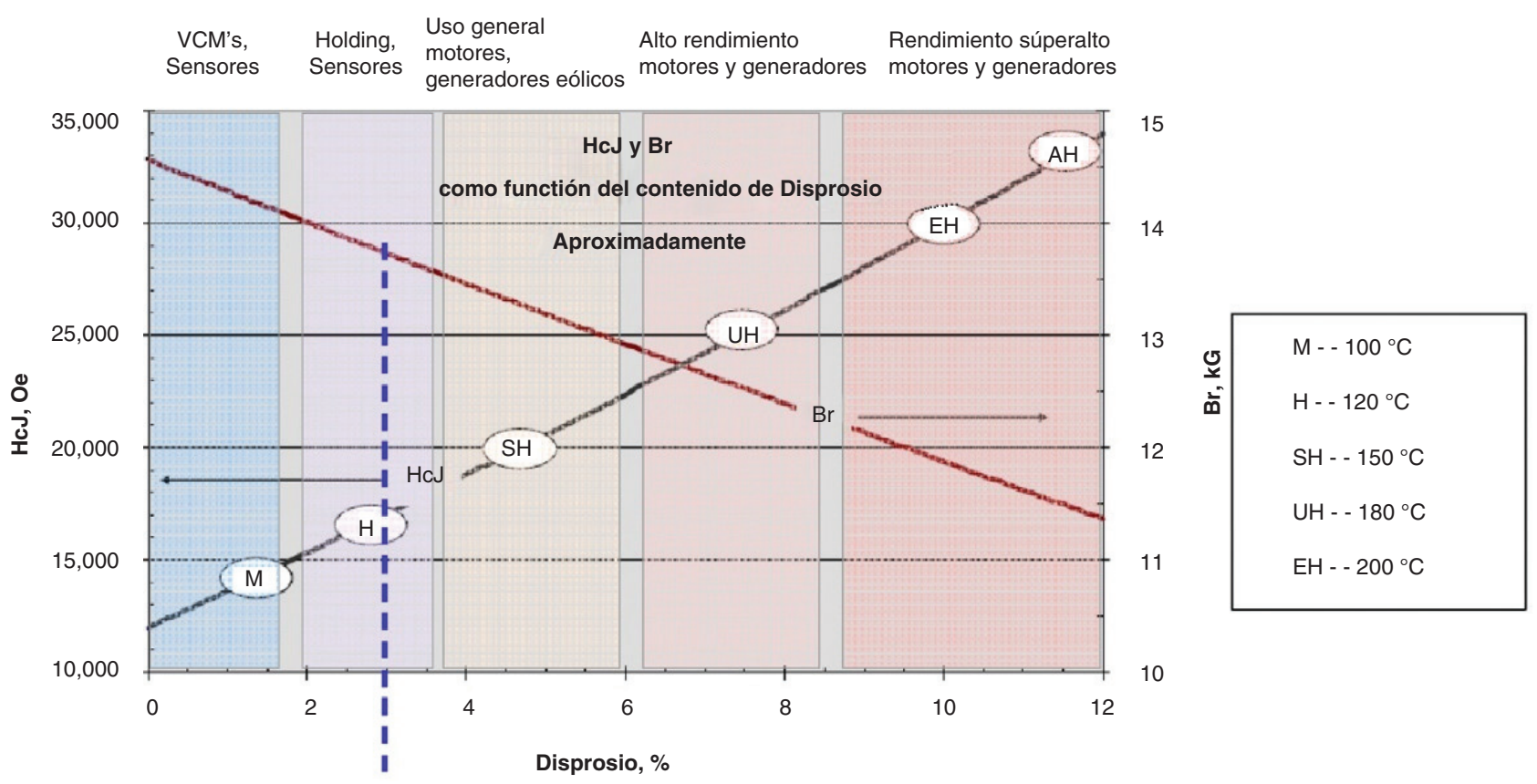

FIGURA 2. Variación de las características magnéticas con la proporción de disprosio (Constantinides, 2010). 


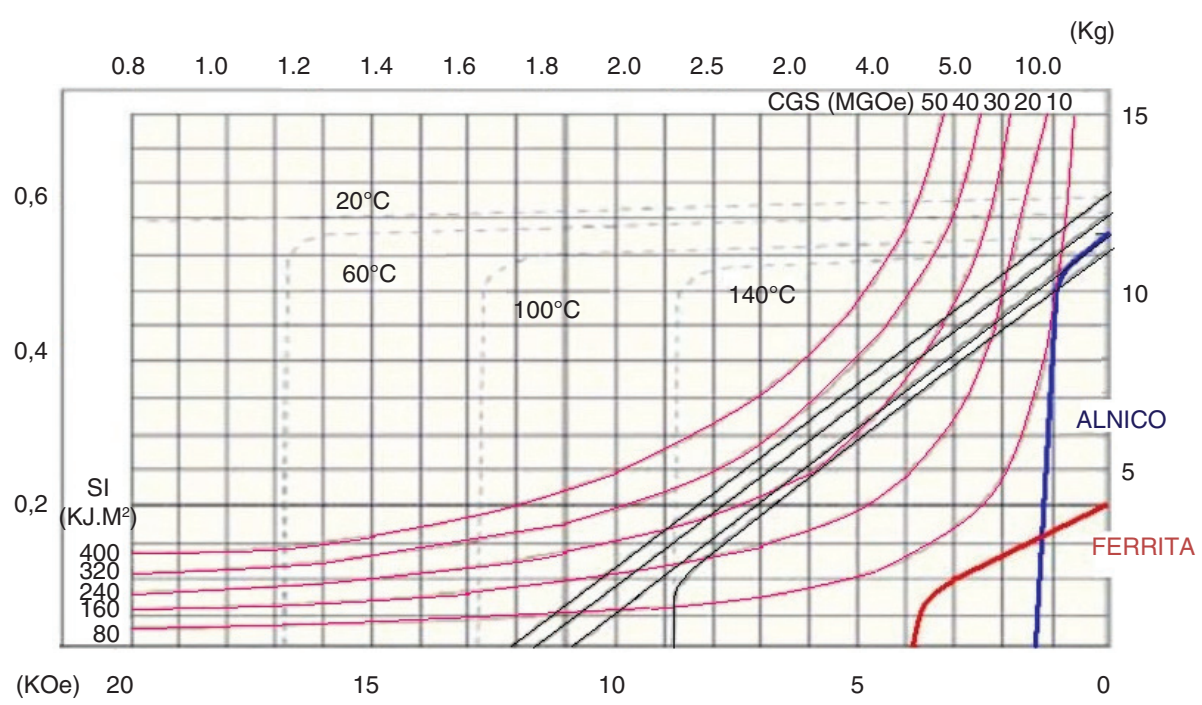

Figura 3. Curvas de desmagnetización típica de N38SH y comparativa con Ferrita y Alnico (Emagnets UK, 2017).

\subsection{Imanes Neo sinterizados o de densidad completa}

Todos los imanes sinterizados son anisotrópicos, lo que favorece sus altos productos de energía alcanzando actualmente valores de 52 MGOe y que es el motivo de ser los elegidos para la construcción de motores eléctricos de hasta $60 \mathrm{~kW}$ para vehículos (Gutfleisch et al., 2011).

Se muestran en la Fig. 3 las curvas comerciales de magnetización del N38SH superpuestas con las de un ALNICO (en azul) y de una ferrita (en rojo), representándolas en la misma escala para poder compararlas.

\subsubsection{Procesos de fabricación de imanes sinterizados}

Los imanes de neodimio sinterizados se preparan fundiendo las materias primas en un horno, colando el material fundido en moldes y enfriándolo para formar lingotes. Luego se produce un polvo extremadamente fino a partir de estos lingotes. Para ello se utiliza la decrepitación de hidrógeno, que se comenta más adelante. Este polvo es sometido más tarde a un proceso de sinterizado en fase líquida, por medio del cual las partículas se alinean magnéticamente y se unen entre sí para formar bloques densos, los cuales son tratados térmicamente. Posteriormente son cortados con la forma deseada y, sometidos a un tratamiento superficial para prevenir la corrosión, y finalmente magnetizados mediante la exposición a un fuerte campo magnético creado mediante bobinas.

Actualmente la tecnología de fabricación de estos imanes está bien establecida (ver Fig. 4) y los costos del proceso son moderados. Como aglutinante se emplean elastómeros como caucho nitrilo, polietileno y vinilo y no suele superar un $5 \%$ de la composición total.

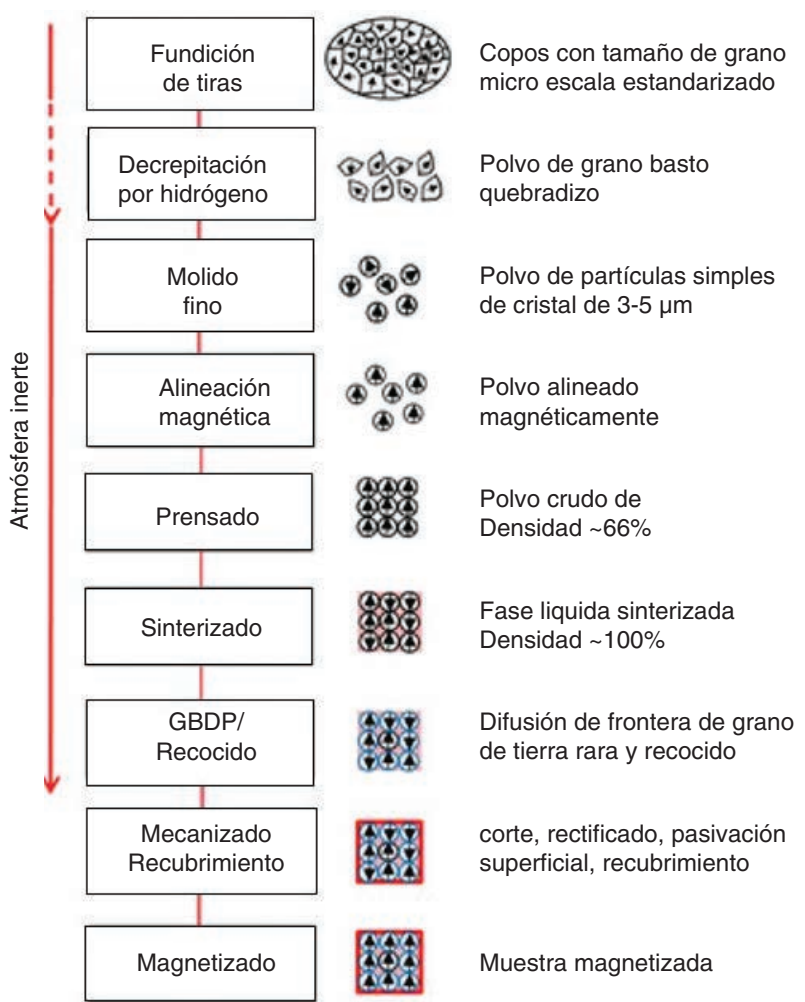

Figura 4. Proceso esquemático de manufactura de imanes sinterizados (Yang et al., 2017).

\subsubsection{Principales variaciones en los procesos de fabricación de imanes NEO}

3.1.2.1. Proceso de sinterizado. Una de las mejoras más fáciles de adoptar se refiere al proceso combinado de sinterizado y alineado, con diversas 
combinaciones posibles. El método más básico incorpora un campo de alineación axial como se muestra en la Fig. 5a. Las flechas negras indican el sentido de prensado y las flechas azules el de magnetización. Una simple bobina toroidal rodea el recipiente para proporcionar un campo relativamente uniforme dentro de él, mientras que en la Fig. 5c se comprime isostáticamente en dos sentidos.

3.1.2.2. Hidrogenación - Decrepitación (HD). Cuando se inyecta hidrogeno en un lingote de imán, los metales de transición y las tierras raras se vuelven frágiles después de su absorción. Al calentarlo se produce una gran expansión de volumen de la retícula del metal que con la ebullición facilita la rotura y permite ser molidos en forma de polvo. Con este método se refina el tamaño del grano de $100-500 \mu \mathrm{m}$ a $0,3 \mu \mathrm{m}$. Este procedimiento en ocasiones se desarrolla en una atmosfera inerte (habitualmente de argón) para evitar la oxidación. La fase rica en neodimio facilita la absorción de hidrogeno a temperatura ambiente, en cambio cuando se emplea la fase estequiométrica es necesario calentar hasta los $150{ }^{\circ} \mathrm{C}$.

Aunque esta técnica ha mejorado algunos de los parámetros magnéticos, se le pueden achacar también inconvenientes como el empeoramiento de la estabilidad térmica y la complejidad propia del proceso, con reacciones exotérmicas y endotérmicas, lo que ha limitado en parte la expansión comercial de esta técnica. A principios del 2000 Aichi Steel retomó

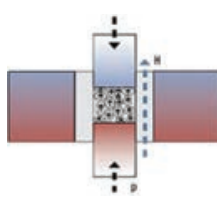

a) Alineamiento Axial

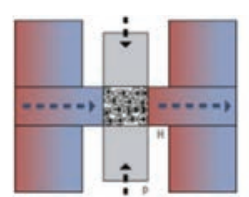

b) Alineamiento transversal

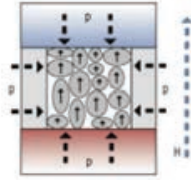

c) Presado isostático con polvo pre-allineado
FIgurA 5. Posibilidades de prensado en fabricación de imanes permanentes (Anderson, 2012a).

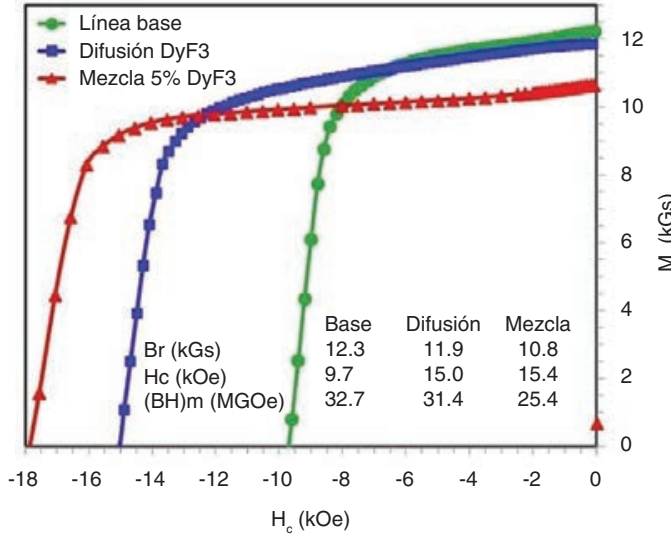

este proceso, mejorándolo y consiguiendo uno de los polvos aglomerados de mayor energía del mercado.

\subsubsection{Incorporación de Dy y sustitución por} $D y F 3$. Como se ha indicado, para poder soportar las temperaturas de funcionamiento de estos imanes en los motores sin que disminuya drásticamente su valor de coercitividad se añade disprosio, lo que aumenta la anisotropía del material y provoca también el aumento la $H_{C}$ y $\beta$ (ver Fig. 6). La adición de disprosio mejora en gran manera la coercitividad, ya que su momento magnético se acopla en antiparalelo con el de hierro, siendo de nuevo necesario que la proporción sea la apropiada para que no se produzca un efecto adverso de disminución.

Actualmente el contenido de disprosio alcanza valores del $10 \%$, un valor demasiado elevado dado de la rareza del material y el precio que ha alcanzado.

Inicialmente el disprosio se mezclaba molido con los polvos del imán y después se difundía. El método que mejores resultados está proporcionando para disminuir la proporción de disprosio es el denominado GBDP (Grain Boundary Difusion Process) (Anderson, $2012 b)$. En él se introduce $\mathrm{DyF}_{3}$ en forma de pintura basada en etil-alcohol y se calienta a $900{ }^{\circ} \mathrm{C}$. De esta forma se difunde hacia el interior del imán hasta las fronteras de grano. El ahorro de disprosio con este método puede alcanzar hasta un $20 \%$.

Otras posibilidades para mejorar $H_{c}$ sin utilizar Dy son:

a) Refinamiento de grano. Al disminuir el tamaño del grano se disminuye exponencialmente la posibilidad de multidominio. (Sugimoto, 2011; Salazar et al., 2016)

b) Control del dominio. El otro método se basa en el recubrimiento de la pared del grano con la fase Nd-rica que elimina los posibles defectos que puedan aparecer en la superficie del mismo, evitando así los dominios en retroceso.

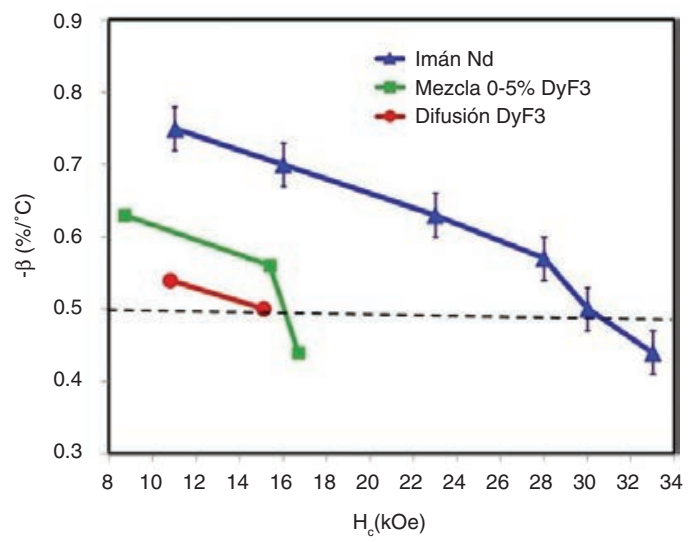

FIgura 6. Comparativa del proceso tradicional-mezcla-difusión (Anderson, 2012b). 


\subsection{Imanes Neo aglomerados (Bonded)}

Estos imanes tienen la misma constitución que los anteriores, con la diferencia de que los granos finos de tierras raras que constituyen el imán permanente se obtienen por procesos de templado muy rápidos obteniendo polvo isotrópico, que se mezclan con una determinada proporción de aglomerante entre un $20-40 \%$, formando una estructura reticular. Posteriormente se constituye el imán por un proceso de compresión o de inyección. También son conocidos como de solidificación rápida.

Inicialmente estos compuestos se habían relegado a un segundo plano, porque ofrecen un flujo magnético menor que el de los imanes sinterizados, sin embargo, en los últimos años el interés por ellos ha crecido, especialmente en aquellas aplicaciones que tiene que ver con los motores de imanes permanentes.

Entre las ventajas que presentan cabe destacar:

- La gran versatilidad que permite en sus procesos de fabricación ha conseguido aumentar el valor del producto, de tal forma que bajo determinadas condiciones son competitivos respecto a los sinterizados.

- Pueden ser moldeados en formas muy intrincadas para obtener la potencia específica requerida, constituyendo polos con formas circulares, incluso se emplean en diseños del tipo multicapa. El polvo obtenido en la actualidad es tan fino que permite mayor grado de compactación.

- Como es mayor la cantidad de aglomerante que incorporan se minimizan las perdidas Joule y corrientes de Foucault en el material magnético y se solucionan los problemas de oxidación sin que sea necesario recubrir con una capa protectora.

- En general los costes de fabricación son menores siempre que hablamos de producción elevada. $\mathrm{Si}$ además se eliminan los elevados costes de montaje que conllevan los prismas, las cuentas a favor de los aglomerados empiezan a salir ya que permiten magnetizarlos después de que el rotor esté ensamblado.

\subsubsection{Procesos de fabricación de imanes aglomerados}

3.2.1.1. Hilado por fusión (Melt Spinning). Los imanes de neodimio aglomerados se preparan a partir de una delgada cinta de aleación de Nd-Fe-B obtenida por medio de hilado por fusión (Grieb et al., 2009). La cinta se pulveriza para producir pequeñas partículas, que son mezcladas con un polímero con una proporción que varía entre el 25 y $40 \%$. La hilatura por fusión es el método comercialmente más empleado debido a su amplia gama de velocidades de enfriamiento y por la capacidad para suministrar microestructuras uniformes en una variedad de aleaciones.

Sin embargo, las principales deficiencias de esta técnica de procesamiento surgen de la forma del producto solidificado. Los copos obtenidos, por su geometría, reducen el factor de relleno y aumentan la viscosidad

3.2.1.2. Atomización por gas (HPGA). Se pensó que el procesamiento mediante atomización de gas a alta presión (High Pressure Gas Atomization) podría llegar a convertirse en un sustituto beneficioso del hilado por fusión en la producción de partículas nanocristalinas de $\mathrm{NdFeB}$. El gas, habitualmente helio, se introduce con una velocidad de Mach 2-3, lo que provoca un cizallamiento muy energético de la corriente de masa fundida, obteniéndose partículas finas y esféricas en vez de los copos obtenidos por hilado por fusión (Anderson et al., 2003)

\section{- Recubrimiento de partículas en HPGA}

Las pérdidas magnéticas irreversibles debido a la oxidación y corrosión son un inconveniente importante de este tipo de polvos magnéticos. Los medios más habituales para evitarlas consisten en aplicar recubrimientos de níquel-plata con un espesor de capa entre 10 y $15 \mu \mathrm{m}$.

El siguiente paso ha sido incorporar el proceso de fluoración en la formación de los polvos. La capacidad de recubrir las partículas mientras están "en vuelo" puede eliminar la necesidad de tratamiento posterior a la atomización.

Los últimos procedimientos incluyen la inyección de trifluoruro de nitrógeno $\left(\mathrm{NF}_{3}\right)$ mezclado con un $20 \%$ de argón para conseguir una capa adherente, como muestra la Fig. 7. De este modo mejora aún más el comportamiento de estos materiales frente a la corrosión.

\section{- Variaciones con estos procesos}

Se han realizado varias pruebas de laboratorio variando la composición con adiciones de carburo de titanio y de circonio (TiC, $\mathrm{TiC}-\mathrm{Zr}$ y de $\mathrm{Zr}-\mathrm{ZrC}$ ). También se incorpora cobalto para mejorar su comportamiento con la temperatura. A través de la adición de $\mathrm{Zr}$ como formador de vidrio y de un inhibidor del crecimiento del grano como el Ti, la alta velocidad de enfriamiento que se requiere para formar los copos ya no se convierte en una necesidad, por lo que es posible otro procesamiento con velocidad de enfriamiento menor que la del HPGA.

Se ha probado el procesamiento de una serie de variaciones en la composición de compuestos con los dos métodos indicados anteriormente, son denominados WT (ver Tabla 2) cuando se procesan en hilado por fusión y GA en la modalidad de gas atomizado. Los resultados son los que se muestran 


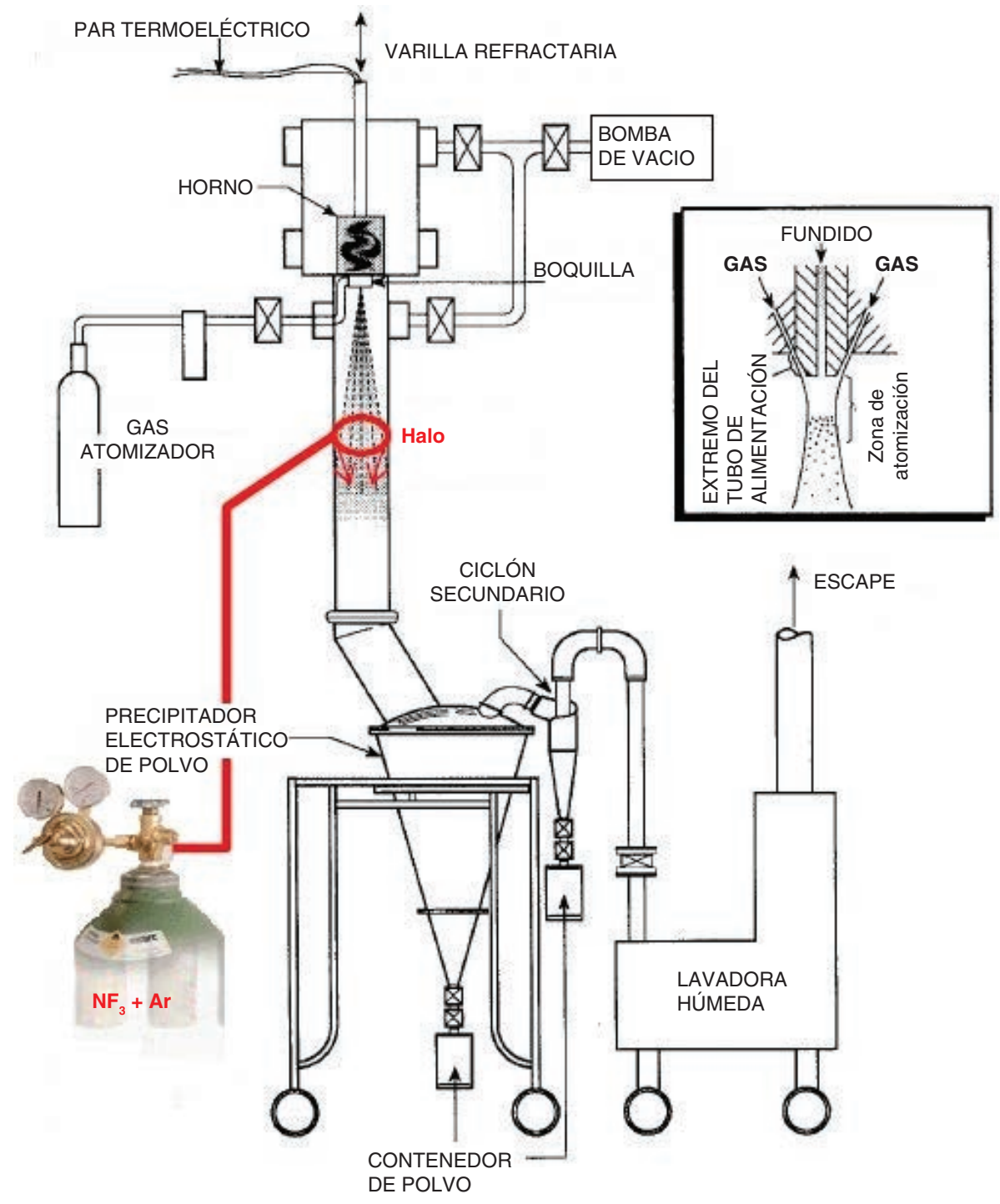

Figura 7. Proceso HPGA con $\mathrm{NF}_{3}$ (Anderson et al., 2003).

TABLA 2. Variación de las composiciones de Ti y Zr

\begin{tabular}{lll}
\hline Hilado por fusión & HPGA & \multicolumn{1}{c}{ Fórmula } \\
\hline WT-096 & GA-1-52 & {$[\mathrm{Nd} 0.45(\mathrm{Y} 0.66, \mathrm{Dy} 0.33) 0.55] 2-0.1 \mathrm{Zr} 0.1(\mathrm{Fe} 0.89, \mathrm{Co} 0.11) 14 \mathrm{~B}+\mathrm{Ti} 0.02 \mathrm{C} 0.02$} \\
WT-102 & GA-1-66 & {$[\mathrm{Nd} 0.45(\mathrm{Y} 0.66, \mathrm{Dy} 0.33) 0.55] 2.3-0.1 \mathrm{Zr} 0.1(\mathrm{Fe} 0.89, \mathrm{Co} 0.11) 14 \mathrm{~B}+\mathrm{Ti} 0.02 \mathrm{C} 0.02$} \\
WT-127 & GA-1-100 & {$[\mathrm{Nd} 0.45(\mathrm{Y} 0.66, \mathrm{Dy} 0.33) 0.55] 2.3-0.3 \mathrm{Zr} 0.3(\mathrm{Fe} 0.93, \mathrm{Co} 0.07) 14 \mathrm{~B}+\mathrm{Zr} 0.01 \mathrm{C} 0.01$} \\
WT-131 & GA-1-106 & {$[\mathrm{Nd} 0.45(\mathrm{Y} 0.66, \mathrm{Dy} 0.33) 0.55] 2.3-0.3 \mathrm{Zr} 0.3(\mathrm{Fe} 0.93, \mathrm{Co} 0.07) 14 \mathrm{~B}+\mathrm{Zr} 0.01 \mathrm{C} 0.01$} \\
\hline
\end{tabular}

en la Fig. 8. En la figura, las curvas con marcadores cuadrados son producidas mediante hilado por fusión y los circulares por atomización con gas, los marcadores rellenos representan modelos comerciales frente a los huecos que son de laboratorio.

Se puede comprobar que cuando el tratamiento elegido es Hilado por Fusión, el denominado WT-102, mejora el comportamiento con la temperatura respecto al original MQP 14-12, y todavía son más planas las curvas obtenidas mediante HPGA, pero en cambio el valor de energía a temperatura ambiente es mucho menor (7,6 frente a 11,5 MGOe). Pero aun considerando estas mejoras no se ha obtenido lo esperado con este método. 


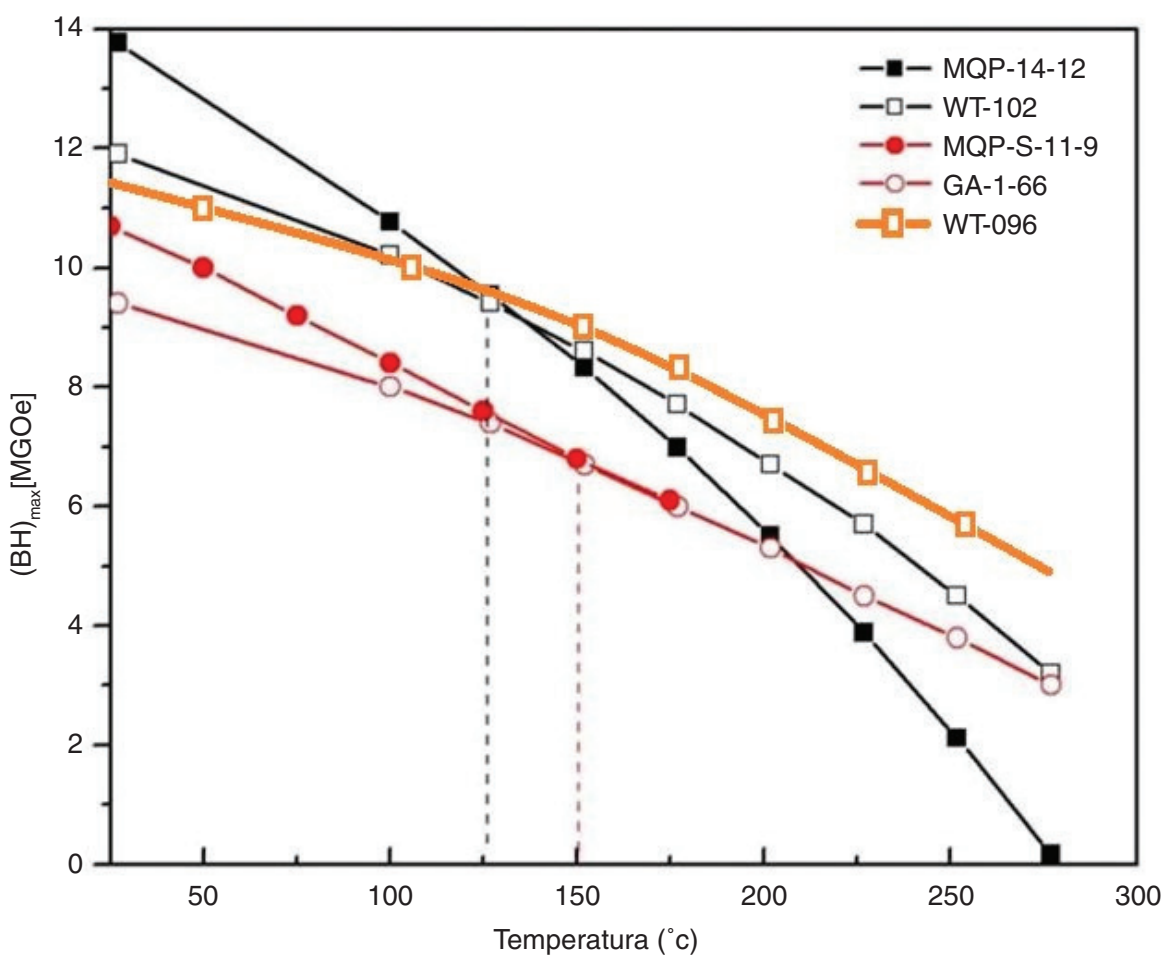

Figura 8. Comparativa de energía entre morfología de copo y esférica (Anderson et al., 2003).

3.2.1.3. MAGFINE C. HDDR (HidrogenaciónDecrepitación-Desorción-Recombinación). E1 método procede del HD empleado con los imanes sinterizados. Fue desarrollado en el año 2000 por Aichi Steel Company, que es la única compañía que lo comercializa, y el nombre proviene de MAGNET (de imán) y FINE (de la microestructura fina) (Aichi, 2012; Honkura, 2013). Existen tres versiones con productos de energía $B H_{\max }: 15,20$ y 25 y es el imán aglomerado con la fuerza magnética más alta. Actualmente el MF-25 alcanza 43MGOe en polvo, que se convierten entre los 23 y los 17 MGOe cuando se moldea por compresión e inyección respectivamente. Presenta una buena resistencia a la temperatura hasta $150{ }^{\circ} \mathrm{C}$ y se puede inyectar directamente en los huecos de los polos de los motores. En el 2014 se han diseñado el primer prototipo para motores auxiliares con una potencia de $50 \mathrm{~W}$.

El proceso HDDR utiliza una serie de cuatro transformaciones dependientes de la temperatura y de la presión en una atmósfera de hidrógeno y controlando la velocidad de absorción-desorción. De este modo se minimiza el tamaño de los cristales y se eleva la anisotropía de los mismos (Ver Fig. 9). El material se descompone en $\mathrm{NdH}_{2}, \mathrm{Fe}$ y $\mathrm{Fe}_{2} \mathrm{~B}$ durante el primer tratamiento térmico cuando se calienta a aproximadamente $840{ }^{\circ} \mathrm{C}$ bajo una presión elevada de gas de hidrógeno.

Durante el siguiente tratamiento es necesario mantener la temperatura entre $750-900{ }^{\circ} \mathrm{C}$ durante
2 - $3 \mathrm{~h}$ con ello el hidrógeno es liberado del hidruro de Neodimio $(\mathrm{NdH})$, lo que se conoce como desorción. Luego el neodimio se recombina con $\mathrm{Fe}_{2} \mathrm{~B}$ para formar el compuesto original, pero con un tamaño de grano más fino, reducido a un tamaño de $0,3 \mu \mathrm{m}$. Estos últimos procesos se realizan en atmosfera de vacío.

La ventaja respecto al tipo de polvo obtenida mediante hilado por fusión es la elaboración directamente de material anisotrópico. El polvo de imán isotrópico presenta curvas con más cuadratura en la curva intrínseca de desmagnetización, lo que le confiere una mayor estabilidad. En cambio, los obtenidos mediante HDDR exhiben curvas más redondeadas. El principal inconveniente que conlleva es su deterioro con la temperatura (hasta un $14 \%$ el MF-15), debido a la poca uniformidad en la distribución del tamaño del grano.

En la tercera generación, MAGFINE ha mejorado este aspecto. Para ello es necesario una nueva etapa con tratamiento de calor adicional para difundir el disprosio en el polvo, que luego se recombina en $(\mathrm{Nd}, \mathrm{Dy})_{2} \mathrm{Fe}_{14} \mathrm{~B}$. Otra de las posibilidades empleadas es mezclar con polvos de $\mathrm{Nd}-\mathrm{Cu}-\mathrm{Al}$, que, al pasar a fase líquida, recubren los granos con un enriquecimiento de neodimio.

$\mathrm{Si}$ bien este proceso HDDR es relativamente complejo, el polvo producido es susceptible de técnicas de conformación y moldeo bastante comunes. Para que sea adecuado para la compresión el polvo 


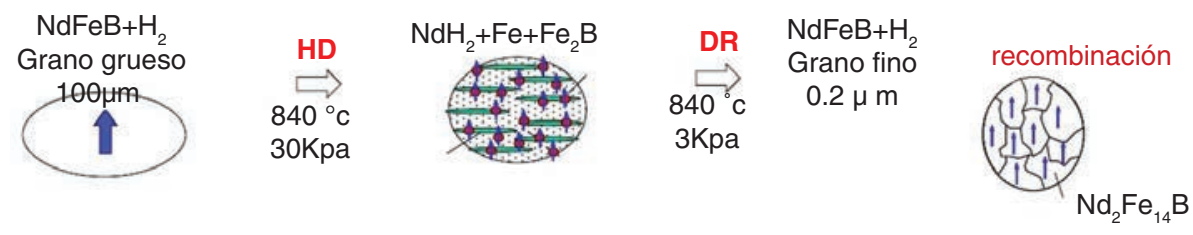

Figura 9. Proceso de fabricación de imanes HDDR (Honkura, 2013).

MF-25 se mezcla con resina epoxi, obteniendo un compuesto termoestable que puede ser moldeado mediante prensado, que además se oxida menos que con otros aglomerantes.

El uso de MAGFINE permitirá reducir el peso y tamaño de un motor considerablemente. De hecho, es el único material que consigue cumplir los objetivos de potencia y densidad específica de motores establecidos por el Advanced Power Electronics and Electric Motors (APEEM) para el año 2020, sin que en su composición se incluya el cotizado disprosio.

3.2.1.4. Deformación en caliente. En la carrera contra la compañía japonesa Aichi por alcanzar las mejores propiedades de estos imanes, la americana Molycorp, desarrolla esta técnica emergente de deformación en caliente directamente de los copos, obteniendo su producto comercial denominado MAGNEQUENCH MQU ${ }_{T M}$.

Los copos se obtienen mediante un proceso de calandrado. El polvo de imán se derrite previamente a $260{ }^{\circ} \mathrm{C}$ bajo la presencia de un campo magnético axial de $20 \mathrm{kOe}$ y se somete a altas presiones para evitar las reacciones gas-sólido. De esta forma se obtienen polvos muy anisotrópicos y con una mejora importante en la estabilidad térmica.

Los fabricantes de estos imanes deformed $M Q U^{\mathrm{TM}}-F$ han informado que tienen un producto de energía de 22 MGOe. Mejorar la estabilidad térmica de estos imanes es tan sencillo como incorporar Dy en su composición, consiguiendo así deformed $M Q U^{\text {TM }}-G$ con valores de energía de $17 \mathrm{kOe}$ y temperatura de operación de $120^{\circ} \mathrm{C}$ sin pérdida de flujo (Brown et al., 2002). El coeficiente de estabilidad de temperatura de coercitividad, $\beta$, se consigue reducir de 0,63 en los MAGFINE a los 0,52 en deformed $\mathrm{G}$.

Como conclusión, los imanes de última generación $M Q U_{T M}$ basados en el proceso de deformación en caliente obtienen estabilidad térmica y altos productos de energía.

\subsubsection{Posibilidades comerciales de imanes aglomerados}

En una primera clasificación existen los polvos isotrópicos y los anisotrópicos. Estos últimos acaparan todo el interés, ya que tiene un mayor producto de energía. Existen varios métodos para transformarlos de isotrópicos en anisotrópicos, como ser prensados en un campo de $20 \mathrm{kOe}$ a $135{ }^{\circ} \mathrm{C}$ de temperatura
(Brown et al., 2002). La principal promotora de estos imanes BPM es Magnequench, que los denomina según las siglas:

- MQP-Isotrópicos

- MQPS de grano esférico (HPGA).

- MQPHTP Alta temperatura y presión.

- MQA-Anisotrópicos.

- MQF-Fine particle.

- MQU-Hot deformed.

Algunos de los comercialmente más conocidos se muestran en la Tabla 3 y su curvas de magnetización en la Fig. 10.

\subsection{Variaciones de la composición química}

En el siguiente apartado se analizan los posibles cambios en las composiciones químicas de los imanes neo (tanto sinterizados como aglomerados) que se están investigando actualmente.

\subsubsection{Progreso en los compuestos MRE-2-14-1}

Con el objetivo de mantener los valores magnéticos, especialmente un $B H_{\max }$, adecuado en las temperaturas de operación; se propone una primera modificación con una mezcla de tierras raras MRE-Fe-B (MRE = Nd, Y, Dy) en la proporción adecuada (Anderson et al., 2003; Sokolowski, 2007). El estudio realizado con los componentes anteriores indica que, respecto al momento magnético, la curva de neodimio presenta la máxima remanencia y la energía anisotrópica hace se hace máxima con el uso de disprosio. Este mix de tierras raras, en los márgenes de temperatura de funcionamiento del motor, mejora la media del parámetro de energía, pero en cambio disminuye a temperatura ambiente.

3.3.1.1. El cobalto. Con pequeñas sustituciones de cobalto por hierro $\left(\left[\mathrm{Nd}_{\mathrm{x}}(\mathrm{YDy})_{0.5(1-\mathrm{x})}\right]_{2.2} \mathrm{Co}_{\mathrm{yFe} 12.5(1-\mathrm{y})}\right.$ B) se mejora sustancialmente la $\mathrm{T}^{\mathrm{a}}$ de Curie. Se observa que con sustituciones entre 0,1 al 0,3 la mejora es muy importante. A partir de ese valor, la curva pierde pendiente. A cambio se produce una disminución de la coercitividad intrínseca (Sokolowski, 2007). Por tanto, hay que buscar una sustitución adecuada de cobalto y disprosio que minimice los efectos adversos y mejore la estabilidad térmica. 
Evolución y proceso de fabricación de imanes "NEO" aplicados a motores de vehículos eléctricos • 11

TABla 3. Materiales magnéticos de Magnequench (Brown et al., 2002)

\begin{tabular}{lll}
\hline Código & \multicolumn{1}{c}{ Composición } & \multicolumn{1}{c}{ Característica } \\
\hline MQPTM-14-12 & RE-Fe-B- & Material nanoestructurado hilado por fusión isotrópico termoestable \\
MQPTM-16-7 & RE-Fe-B-type & Material nanoestructurado hilado por fusión isotrópico de alta remanencia \\
Deformado F MQU & Nd-Fe-Co-B-Ga & Material hilado por fusión con alta remanencia deformado en caliente \\
Deformado G MQU & Nd-Dy-Fe-Co-B-Ga & Material hilado fundido con alta coercitividad deformado en caliente \\
MF20/ MF15 AICHI Acero & Nd-Fe-B-Nb-Ga & Material procesado HDDR anisotrópico \\
MQPTM-A-T & Nd-Fe-Co-B-Ga & Material original con nuevo proceso HDDR desarrollado en la década de 1990 \\
\hline
\end{tabular}

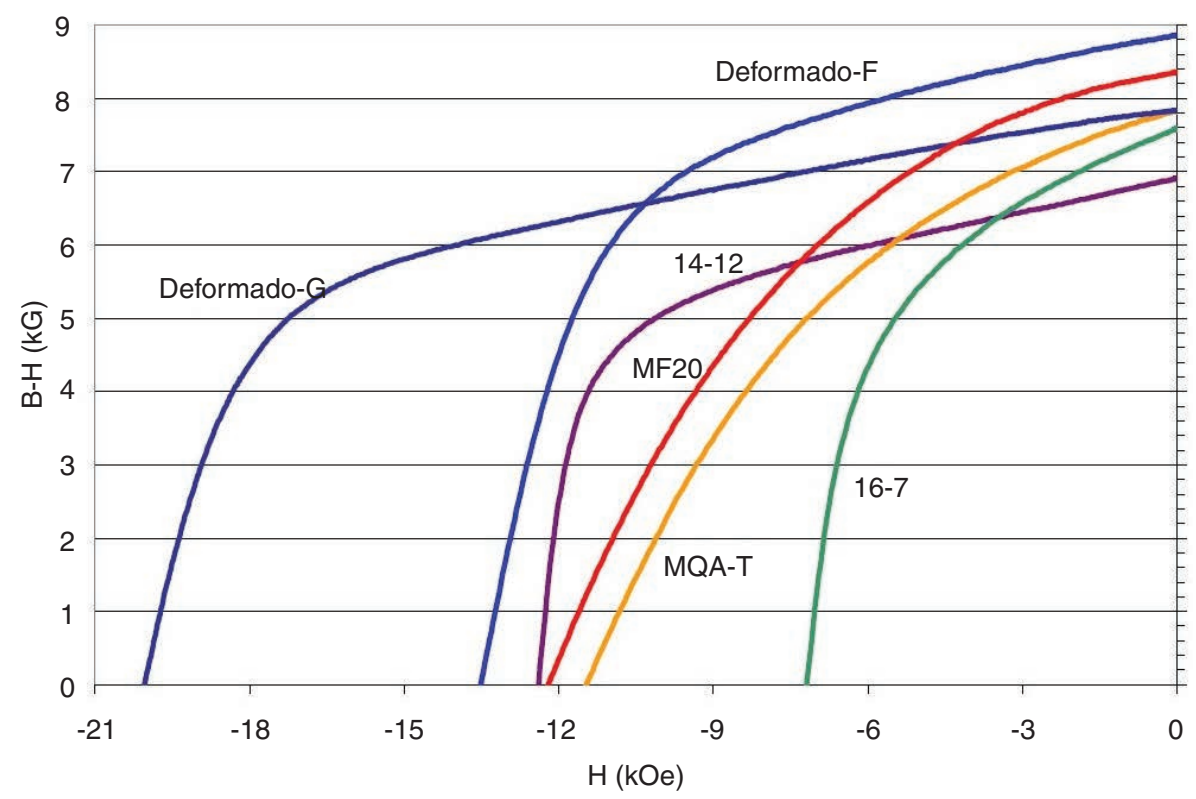

Figura 10. Comparativa de los distintos imanes aglomerados según proceso de fabricación (Brown et al., 2002).

En el análisis en (McCallum, 2012), en vez de sustituir el cobalto por hierro, se sustituye una mezcla de tierras raras $\left(\left[\mathrm{Nd}_{\mathrm{x}}(\mathrm{YDy})_{0.5(1-\mathrm{x})}\right]_{2.2-\mathrm{y}} \mathrm{Co}_{\mathrm{y}} \mathrm{Fe}_{12.5} \mathrm{~B}\right)$ por cobalto y se observa como que hasta una concentración del 1,5\% aumenta sustancialmente el valor de la energía y disminuye muy levemente la coercitividad. A partir de ese punto la mejora no es sustancial y la coercitividad empieza a disminuir sin aumentar el valor de $\mathrm{BH}$.

3.3.1.2. El zirconio. Otro de los efectos que presenta la adición de cobalto, así como los enriquecimientos de neodimio es el incremento del crecimiento de las fases suaves $\alpha-F e$, para contrarrestar este efecto se añade circonio que es bien conocido por su capacidad para formar cristales de vidrio. Inhibe eficazmente el crecimiento de granos durante la solidificación, impide la nucleación de fases suaves y promover la formación de la fase dura 2-14-1.

Además, disminuye la viscosidad de los copos y facilita las operaciones de prensado y por tanto reduce sustancialmente los costes de manufactura finales. El valor óptimo se logró mediante la adición de $0,4 \%$ en peso de zirconio.

3.3.1.3. El carburo de titanio (TiC). No tiene solubilidad en el MRE2-14-1, formando precipitados en los límites del grano. Tiene una función similar a la de circonio Estos precipitados proporcionaron un lugar de nucleación para la fase magnético duro, así como un mecanismo eficaz que impida el crecimiento de grano durante el recocido, por lo que se consigue una microestructura refinada. El conjunto de zirconio y $\mathrm{TiC}$ proporciona cristalizaciones uniformes.

3.3.1.4. La plata. Otra de las mejoras introducidas reducir la velocidad de solidificación y usar aditivos muy solubles como plata. Se han hecho pruebas incorporando hasta el $0,3 \%$, la proporción óptima es del $0,1 \%$ presentando buenos resultados tanto en su comportamiento ante la temperatura, como ante la oxidación. 
3.3.1.5. Una nueva posibilidad: El cerio. Con el objetivo "No es el mejor imán de tierras raras, pero mucho mejor que cualquier imán sin tierras raras", se pretende diseñar un nuevo compuesto en el que el material principal sea el Cerio.

Los motivos por los que se ha elegido este elemento es que es una tierra rara muy abundante con una estructura y distribución electrónica interesante. Es un elemento que tiene una valencia entre 4 y 3 , cuanto más se aproxime a la valencia 3 mejor consigue una anisotropía estructural o magnetocristalina (Zhao et al., 2017), siendo la fórmula propuesta es $\left(\mathrm{Ce}_{1}-\mathrm{xNdx}\right)_{2} \mathrm{Fe}_{14} \mathrm{~B}$.

Las modificaciones en las composiciones químicas propuestas que influyen directamente sobre la estructura atómica de los imanes se dirigen a aumentar la vida útil y reducir costes, tanto de imanes sinterizados como en aglomerado, manteniendo el valor del producto de energía en valores deseables.

\subsection{Imanes aglomerados frente a sinterizados}

Una de las aplicaciones de imanes aglomerados en desarrollo es la sustitución de los imanes sinterizados en motores eléctricos, especialmente los síncronos de imanes interiores, pues con los imanes sinterizados las geometrías se limitan a líneas rectas principalmente y éstos deben construirse, magnetizarse, mecanizarse y tratarse superficialmente antes de su montaje. Los imanes aglomerados, que además son más ligeros, pueden inyectarse directamente en los alojamientos preparados en el rotor y magnetizarlos in situ. La geometría puede ser entonces más compleja, proporcionando un mayor par motor y se busca un compromiso entre un menor producto de energía de los aglomerados y un par razonablemente alto para poder sustituir los imanes sinterizados.

En un trabajo realizado por los autores se ha procedido a simular en condiciones muy similares mediante elementos finitos el motor MG2 del Toyota Prius 2004 (motor síncrono de imanes permanentes con imanes sinterizados N36) en los que la geometría de los imanes es plana en $\mathrm{V}$ y de otros modelos en los que se consideran imanes curvos y se disponen en doble capa. Este modelo doble curvo se ha simulado con los mismos imanes sinterizados (N36) y con imanes aglomerados (NH-10). Como muestran las curvas de la Fig. 11, la geometría doble curvo con imanes sinterizados proporcionaría un par magnetostático muy superior al de la geometría plana, alcanzando un máximo que alcanza los $900 \mathrm{Nm}$, mientras que con imanes aglomerados ronda los $600 \mathrm{Nm}$ que aun así es muy superior al del Toyota Prius. Pero la conformación del diseño doble curvo con sinterizados no es factible.

\section{CONCLUSIONES}

- El presente documento ha pretendido revisar los avances y variaciones que se han producido en los últimos tiempos tanto en las técnicas metalúrgicas para la obtención del polvo magnético, así como en la composición de los mismos analizando los efectos en las propiedades de los mismos.

- Cada día se demanda más alguna investigación que ofrezca alguna alternativa interesante a este tipo de imanes, aunque no se conseguido un reemplazamiento total, sí que las investigaciones descritas han permitido una disminución sustancial de los materiales más críticos, permitiendo alcanzar potencias para los aglomerados cada vez más próximas a los sinterizados que sumándolo a la flexibilidad que permiten en las formas compensa sobradamente la escasa diferencia en el producto de energía que presentan.

- Además, los aglomerados permiten un montaje más sencillo, magnetización una vez colocados junto con menores pérdidas y mejor

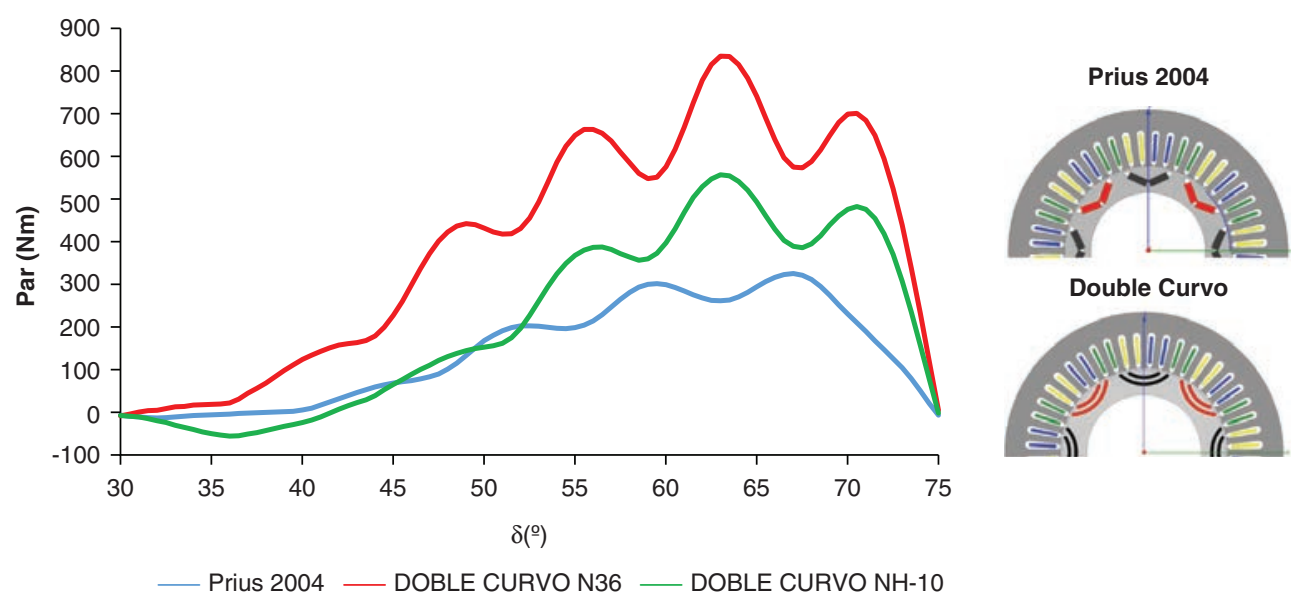

Figura 11. Curvas de par magnetostático para el MG2 del Prius 2004 y para el modelo doble curvo, con sinterizados (N36) y aglomerados (NH-10). 
comportamiento ante la corrosión. Aunque la cantidad total de material a emplear para una potencia específica dada $(2,88 \mathrm{~kW} / \mathrm{kg})$ sea algo mayor $1.39 \mathrm{~kg}$ frente a $0,65 \mathrm{~kg}$, el coste total de los manes es más reducido.

- $\quad$ Por todo esto los aglomerados están ampliando el abanico de posibilidades a motores de mayor potencia y apostamos a que sin otro descubrimiento por el momento serán el material para los nuevos vehículos. Aun así, las condiciones económico políticas han impulsado que compañías como Tesla y Renault utilicen motores de inducción.

\section{REFERENCIAS}

Abad, V., Sagredo, J. (2018). Imanes permanentes de tierras raras. El nuevo oro negro de los vehículos eléctricos. Revista DYNA 93 (1), 47-51.

Aichi Steel Company (2012). MAGFINE Technical Datasheet. Disponible Web 10/07/2018: https://www.aichi-steel.co.jp/ ENGLISH/products/electromagnetic/bonded_magnet/ item/magfine_datasheet-201705.pdf.

Anderson, I.E., McCallum, R.W., Kramer, M.J. (2003). Development of improved Podwer for Bonded Permanent Magnets. IEEE International Magnetics Conference (INTERMAG), Boston, USA. https://doi.org/10.1109/ INTMAG.2003.1230684.

Anderson, I.A. (2012a). Permanent Magnet Development for Automotive Traction Motors. Hydrogen and Fuel Cells Program and Vehicle Technologies. AMES Laboratories. Disponible Web 10/07/2018: https://www.energy.gov/sites/ prod/files/2014/03/f10/ape015_anderson_2012_o.pdf.

Anderson, I.A. (2012b). Accomplishments in Rare Earth Anisotropic (R2Fe14B-type) Magnet Research. FY 2011 Progress Report. A.P.E. a. E. Motors. Disponible Web 10/07/2018: https://www.energy.gov/sites/prod/files/2014/03/f8/2011_ apeem_report.pdf.

Boldea, I., Tutelea, L.N., Parsa, L., Dorrell, D. (2014). Automotive Electric Propulsion Systems with Reduced or No Permanent Magnets: An Overview. IEEE T. Ind. Electron. 61 (10), 5696-5711. https://doi.org/10.1109/TIE.2014.2301754.

Brown, D.N., Na, B.-M., Campbell, P. (2002). The comparison of anisotropic (and isotropic) powders for polymer bonded Rare-Earth permanent magnets. International Workshop; 17th, Rare-Earth Magnets and their Applications, Newark, USA, pp. 62-73.

Constantinides, S. (2010). The Magnetic Material Challenge. ARPA-E Workshop Rare Earth and Critical Materials. Arlington, VA. Disponible Web 10/07/2018: https://www. yumpu.com/en/document/view/37661052/the-magneticmaterial-challenge-arnold-magnetic-technologies.

DOE (2011). Critical materials Strategy. Department of Energy USA. DOE/PI/0009. https://www.energy.gov/sites/prod/ files/DOE_CMS2011_FINAL_Full.pdf.

Deshpande, U.S. (2003). Recent advances in materials for use in permanent magnet machines-a review. IEEE International Electric Machines and Drives Conference, IEMDC'03, Vol 1, pp. $509-515$.

Emagnets UK (2017). Temperature effects on alnico magnets. Disponible Web 10/07/2018: http://e-magnetsuk.com/alnico_ magnets/temperature ratings.aspx.

Fessler, R. (2011). Final Report on Assessment of Motor Technologies for Traction Drives of Hybrid and Electric
Vehicles. Oak Ridge National Laboratory. Disponible Web 10/07/2018: https://info.ornl.gov/sites/publications/files/ pub28840.pdf.

Grieb, B., Brown, D., Miller, D., Shet, N. (2009). Cost effective motor design, Based on: Isotropic and Anisotropic bonded $\mathrm{NdFeB}$ magnets. Magnequench, Leading Magnet Innovation. Magnetic Materials in Electrical Machine Applications, Pori, Finlandia. Disponible Web 10/07/2018: http://www.prizz.fi/sites/default/files/tiedostot/linkki2ID416.pdf.

Gutfleisch, O., Willard, M.A., Brück, E., Chen, C.H., Sankar, S.G., Liu, J.P. (2011). Magnetic materials and devices for the 21st century: stronger, lighter, and more energy efficient. Adv. Mater. 23 (7), 821-842. https://doi.org/10.1002/ adma.201002180

Honkura, Y. (2013). The development of Dy free MAGFINE and its applications to Motors. Symposium on "Current Research Trends in Magnetic Materials". http://komag. org/2013winter/Yoshinobu\%20Honkura.pdf.

Keisan, O. (2016). Electric Machines Design Review. Power Lab at Middle East Technical University, Turkey. Disponible Web 10/07/2018: http://keysan.me/presentations/ee361_ magnets.html\#14.

Liu, J. (2010). Rare Earth Magnet Design Considerations. ARPA-E Workshop Rare Earth and Critical Materials, Arlington, USA. Disponible Web 10/07/2018: https://www. arpa-e.energy.gov/sites/default/files/documents/files/Breakout_Session_Magnetics_LowRes.pdf

McCallum, R.W. (2012). Replacing critical rare earth materials in high energy density magnets. Bulletin of the American Physical Society 57 (1), APS Meeting 2012, Boston, Massachusetts. Disponible Web 10/07/2018: http://meetings.aps. org/Meeting/MAR12/Event/165012.

Salazar, D., Martín-Cid, A., Madugundo, R., Garitaonandia. J.S., Barandiaran, J.M., Hadjipanayis, G.C. (2016). Effect of $\mathrm{Nb}$ and $\mathrm{Cu}$ on the crystallization behavior of understoichiometric Nd-Fe-B alloys. J. Phys. D Appl. Phys. 50 (1), 015305. https://doi.org/10.1088/1361-6463/50/1/015305.

Sugimoto, S. (2011). Current status and recent topics of rareearth permanent magnets. J. Phys. D Appl. Phys. 44 (6), 064001. https://doi.org/10.1088/0022-3727/44/6/064001.

Sokolowski, P.K. (2007). Processing and protection of rare earth permanent magnet particulate for bonded magnet applications. Thesis, Master of Science, Iowa State University.

Trout, S. (2015). Permanent Magnets 101. Disponible Web 10/07/2018: https://www.slideshare.net/StanTrout/permanentmagnets 101 troutbrief.

Vaimann, T., Kallate, A., Kilk, A., Belahcen, A. (2013). Magnetic properties of reduced Dy NdFeB permanent magnets and their usage in electrical machines. Proceedings Conference Africon 2013, pp. 1-5. https:/doi.org/10.1109/ AFRCON.2013.6757787.

Yang, Y., Walton, A., Sheridan, R., Güth, K., Gauß, R., Gutfleisch, O., Buchert, M., Steenari, B.-M., Van Gerven, T., Jones, P.T., Binnemans, K. (2017). REE Recovery from End-of-Life NdFeB Permanent Magnet Scrap: A Critical Review. J. Sustain. Metall. 3 (1), 122-149. https://doi.org/ 10.1007/s40831-016-0090-4.

Zhao, L., Yu, H., Guo, W., Zhang, J., Zhang, Z., Hussain, M., Liu, Z., Greneche, J.M. (2017). Phase and hyperfine structures of melt-spun nanocrystalline (Ce1-xNdx) 16Fe78B 6 alloys. IEEE T. Mag. 53 (11), 1800205. https://doi.org/10.1109/ TMAG.2017.2695533.

Zhang, S., Xu, J., Junak, J., Fiederling, D., Sawczuk, G., Koch, M., Schalja, A., Podack, M., Baumgartner, J. (2012). Permanent magnet technology for electric motors in automotive applications. $2^{\text {nd }}$ International Electric Drives Production Conference (EDPC), Nuremberg, Germany. https://doi.org/10.1109/EDPC.2012.6425118. 